\title{
Identification of a set of genes associated with response to interleukin- 2 and interferon- $\alpha$ combination therapy for renal cell carcinoma through genome-wide gene expression profiling
}

\author{
OSAMU MIZUMORI ${ }^{1}$, HITOSHI ZEMBUTSU ${ }^{1}$, YOICHIRO KATO ${ }^{1,4}$, TATSUHIKO TSUNODA ${ }^{2}$, FUYUKI MIYA ${ }^{2}$, \\ TAKASHI MORIZONO ${ }^{2}$, TAIJI TSUKAMOTO ${ }^{3}$, TOMOAKI FUJIOKA ${ }^{4}$, YOSHIHIKO TOMITA ${ }^{5}$, \\ TADAICHI KITAMURA ${ }^{6}$, SEIICHIRO OZONO ${ }^{7}$, TSUNEHARU MIKI $^{8}$, \\ SEIJI NAITO ${ }^{9}$, HIDEYUKI AKAZA ${ }^{10}$ and YUSUKE NAKAMURA ${ }^{1}$
}

\begin{abstract}
${ }^{1}$ Laboratory of Molecular Medicine, Human Genome Center, Institute of Medical Science, The University of Tokyo, Tokyo 108-8639; ${ }^{2}$ Laboratory for Medical Informatics, Center for Genomic Medicine, RIKEN, Kanagawa 230-0045; ${ }^{3}$ Department of Urologic Surgery and Andrology, Sapporo Medical University School of Medicine, Sapporo 060-8556; ${ }^{4}$ Department of Urology, Iwate Medical University, School of Medicine, Iwate 020-8505; ${ }^{5}$ Department of Urology, Faculty of Medicine, Yamagata University, Yamagata 990-9585; ${ }^{6}$ Department of Urology, Faculty of Medicine, The University of Tokyo, Tokyo 113-0033; ${ }^{7}$ Department of Urology, Hamamatsu University School of Medicine, Shizuoka 431-3192; ${ }^{8}$ Department of Urology, Kyoto Prefectural University of Medicine, Kyoto 602-8566;

${ }^{9}$ Department of Urology, Graduate School of Medical Sciences, Kyushu University, Fukuoka 812-8582;

${ }^{10}$ Department of Urology and Andrology, Graduate School of Comprehensive Human Sciences, University of Tsukuba, Ibaragi 305-8577, Japan
\end{abstract}

Received June 29, 2010; Accepted August 9, 2010

DOI: $10.3892 /$ etm.2010.148

\begin{abstract}
Interleukin (IL)-2 and interferon (IFN)- $\alpha$ combination therapy for metastatic renal cell carcinoma (RCC) improves the prognosis for a subset of patients, while some patients suffer from severe adverse drug reactions with little benefit. To establish a method to predict responses to this combination therapy (approximately 30\% response rate), the gene expression profiles of primary RCCs were analyzed using an oligoDNA microarray consisting of 38,500 genes or ESTs, after enrichment of the cancer cell population by laser microbeam microdissection. The analysis of 10 responders and 18 non-responders identified 24 genes that exhibited significant differential expression between the two groups. In addition, the patients whose tumors did not express HLA-DQA1 or HLA-DQB1 molecules demonstrated poor clinical response. Exclusion of patients with tumors lacking either of these two genes is likely to improve the response rate to IL-2 and IFN- $\alpha$
\end{abstract}

Correspondence to: Dr Yusuke Nakamura, Laboratory of Molecular Medicine, Human Genome Center, Institute of Medical Science, The University of Tokyo, 4-6-1 Shirokanedai, Minato-ku, Tokyo 108-8639, Japan

E-mail: yusuke@ims.u-tokyo.ac.jp

Key words: interleukin-2 and interferon- $\alpha$ combination therapy, HLA-DQA1, HLA-DQB1 combination therapy from 30 to $67 \%$, indicating that a simple pretreatment test provides useful information with which to subselect patients with renal cancer in order to improve the efficacy of this treatment and reduce unnecessary medical costs.

\section{Introduction}

Renal cell carcinomas (RCCs) account for 2-3\% of all malignancies $(1,2)$. Surgical resection is the first choice of treatment for RCC at an early stage. However, when patients present at an advanced stage or have local recurrence or distant metastasis to other organs, immunotherapy, chemotherapy and radiation therapy are applied, although the response rates are poor. Recently, new molecular targeted agents, such as sunitinib and sorafenib, have been developed and are widely used (3-7). Although these drugs have demonstrated a better clinical response than previously used treatments, serious adverse reactions, such as fatigue, mucositis, hand-foot syndrome, diarrhea and hypertension, are often observed and become the cause of discontinuation of the drug administration.

Monotherapy or combination therapy of interleukin (IL)-2 and interferon (IFN)- $\alpha$ has been relatively widely applied for the treatment of advanced kidney cancers. In Japan, to reduce the risk of adverse reactions, the dose of IL-2 is lower than that used in other countries (8-11). While the response rate of monotherapy is as low as $10-20 \%$, that of combination therapy is slightly better, $20-25 \%$ (12-17). However, the rate of adverse reactions associated with this combination therapy appears to 
be lower than recently developed molecular targeting drugs $(18,19)$. Hence, we aimed to define a subset of patients who expect to show a favorable response to this therapy through gene expression profiles of metastatic RCCs, after enrichment of the cancer cells with laser microbeam microdissection technology. In the present study, two antigen-presentation-associated molecules were identified that may predict response to IL-2 and IFN- $\alpha$ combination therapy for metastatic RCC. In addition, this finding may be useful for improving the drug response rate, for contributing to the improvement of the quality of life and prognosis of patients, and reducing unnecessary medical costs to non-responders.

\section{Materials and methods}

Patients and tissue samples. Tissue samples from surgically resected RCC and corresponding clinical information were obtained from 21 hospitals (Tokyo University, Okayama University, Sapporo Medical University, Kobe University, Nihon University, Kanazawa University, Isezaki City Hospital, Shinshu University, Kyushu University, Kyoto Prefectural University of Medicine, Osaka Medical Center for Cancer and Cardiovascular Diseases, Hamamatsu Medical University, Sendai Social Insurance Hospital, Iwate Medical University, Okayama Medical Center, Nagoya City University, Tokushima University, Gifu Prefectural General Medical Center, Tokyo Medical University, Tokyo Medical and Dental University, and Tokyo Women's Medical University Medical Center East, Japan) after each patient provided written informed consent. A total of 42 cancer samples (11 women and 31 men; median age 62.5 years; range 25-75) (Table I) that had been histologically confirmed as RCC, were selected for this study. The clinical stage of each patient was assessed according to the Union International Centre Cancer tumor node metastasis classification. Corresponding normal tissue was also obtained from the distant region of the cancer lesion in the resected kidney tissue. These samples were immediately embedded in TissueTek OCT compound (Sakura, Tokyo, Japan), frozen and stored at $-80^{\circ} \mathrm{C}$. The frozen tissues were sliced into $8-\mu \mathrm{m}$ sections using a cryostat (Sakura) and then stained with $\mathrm{H} \& \mathrm{E}$ for histological examination.

Preparation and analysis of RNA. Total RNA purified using the RNeasy Micro kit (Qiagen, Germany) was quantified in a Nano Drop spectrophotometer (ND 1000). The RNAs, whose ratios of OD 260/280 $\mathrm{nm}$ were between 1.7 and 2.0, were used for further analysis.

Microarray analysis. According to the protocol of the T7-Oligo(dT) Promoter Primer kit and IVT Labeling kit (Affymetrix), RNAs were reversely transcribed using oligo(dT) promoter primers with a $\mathrm{T} 7$ recognition site in the first-strand cDNA synthesis. Following purification of the product of the double-strand cDNA after second-strand cDNA synthesis, in vitro transcription (IVT) reaction of biotinylated complementary RNA was carried out using T7 RNA polymerase. Labeled RNA was fragmented and hybridized to the array named Affymetrix Human Genome U133 Plus 2.0. The arrays were washed, stained and scanned using the GeneChip $30007 \mathrm{G}$ scanner (Affymetrix). Signal intensities and the absolute call dataset were generated with Affymetrix Gene Chip Operating Software (AGCC) using the MAS5.0 algorithm.

Identification of genes associated with the clinical response to the treatment. Treatment responses, in detail, evaluated according to the Response Criteria for Urological Cancer Treatment (20), which are nearly identical to the WHO criteria (21), are documented in Tables I and II. We applied a random permutation test to identify genes whose expression levels were significantly different between the responder (clinical response: $\mathrm{CR}$, complete response or $\mathrm{PR}$, partial response) and non-responder groups (MR, minor response; $\mathrm{NC}$, no change or $\mathrm{PD}$, progressive disease); favorable response or poor response was defined by the tumor shrinkage rate of pulmonary metastasis.

The mean $(\mu)$ and standard deviation $(\sigma)$ were calculated from the log-transformed relative expression ratios of each gene in responder (r) and non-responder (n) cases. A discrimination score (DS) for each gene was defined as follows: DS = $\left(\mu_{\mathrm{r}}-\mu_{\mathrm{n}}\right) /\left(\sigma_{\mathrm{r}}+\sigma_{\mathrm{n}}\right)$.

We performed permutation tests to evaluate the ability of individual genes to distinguish between 10 samples in the responders and 18 samples in the non-responders; samples were randomly permutated between the two groups at 1,000,000 times. Since the DS dataset of each gene showed a normal distribution, we calculated a P-value for the userdefined grouping (22).

Quantitative RT-PCR. We identified 24 genes (Table III) that showed significantly different levels of expression between the responder and non-responder groups, based on microarray analysis, and subsequently focused on two immunologically important genes, HLA-DQA1 and HLA-DQB1. To examine the possibility of adapting our prediction system for clinical use, we performed quantitative real-time RT-PCR of the two genes and evaluated their expression levels in all 42 samples by calculating relative expression ratios of each sample. Extracted RNAs were reversely transcribed using the SuperScript VILO cDNA Synthesis kit (Invitrogen) following the supplier's protocol. For the quantification of mRNA levels, real-time quantitative PCR was performed with LightCycler 480 (Roche). The sequences of each primer and probe were as follows: internal control $(\beta 2 \mathrm{M})$, forward primer 5'-TAGGAGGGCTGGCAACTTAG-3'; reverse primer 5'-CCAAGATGTTGATGTTGGATAAGA-3'; and TaqMan Probe 5'-GGGAGCAG-3'; predictive genel (HLA-DQA1), forward primer 5'-ACTATTCTCTGGCCCGGTTT-3'; reverse primer 5'-TACCCCAGGCATGTCTTTGT-3'; and TaqMan probe 5'-CTCCTCCA-3'; predictive gene2 (HLA-DQB1), forward primer 5'-AGCATTTTGGGGTGTCAAGT-3'; reverse primer 5'-ACACAGCACTCACCAAACCA-3'; and TaqMan probe 5'-CAGAGGAG-3'.

PCR reactions were optimized for the number of cycles to ensure product intensity within the logarithmic phase of amplification.

\section{Results}

Laser microbeam microdissection (LMM) was carried out to enrich cancer cell populations from each of 42 tumor tissues 
Table I. Clinicopathological features of patients with renal cell carcinoma.

\begin{tabular}{|c|c|c|c|c|c|c|}
\hline ID & Gender & Age & Histological type & Response & Group & Prediction \\
\hline KIS-020 & M & 63 & Clear cell carcinoma & PR & Responder & Learning \\
\hline KIS-021 & $\mathrm{F}$ & 71 & Clear cell carcinoma & PR & Responder & Learning \\
\hline KIS-028 & M & 71 & Clear cell carcinoma & PR & Responder & Learning \\
\hline KIS-034 & M & 55 & Clear cell carcinoma & PR & Responder & Learning \\
\hline KIS-035 & $\mathrm{F}$ & 74 & Clear cell carcinoma & PR & Responder & Learning \\
\hline KIS-038 & M & 63 & Clear cell carcinoma & PR & Responder & Learning \\
\hline KIS-044 & M & 66 & Clear cell carcinoma & $\mathrm{CR}$ & Responder & Learning \\
\hline KIS-050 & M & 60 & Clear cell carcinoma & PR & Responder & Learning \\
\hline KIS-058 & M & 56 & Clear cell carcinoma & PR & Responder & Learning \\
\hline KIS-061 & M & 65 & Clear cell carcinoma & $\mathrm{CR}$ & Responder & Learning \\
\hline KIS-001 & M & 69 & Clear cell carcinoma & $\mathrm{NC}$ & Non-responder & Learning \\
\hline KIS-005 & M & 25 & $\begin{array}{l}\text { Combined type (cyst-associated and } \\
\text { papillary renal cell carcinoma) }\end{array}$ & $\mathrm{PD}$ & Non-responder & Learning \\
\hline KIS-006 & M & 44 & Clear cell carcinoma & PD & Non-responder & Learning \\
\hline KIS-007 & M & 71 & Clear cell carcinoma & PD & Non-responder & Learning \\
\hline KIS-008 & M & 67 & Clear cell carcinoma & PD & Non-responder & Learning \\
\hline KIS-016 & $\mathrm{F}$ & 67 & Clear cell carcinoma & $\mathrm{NC}$ & Non-responder & Learning \\
\hline KIS-026 & M & 64 & Clear cell carcinoma & $\mathrm{NC}$ & Non-responder & Learning \\
\hline KIS-032 & M & 60 & Clear cell carcinoma & $\mathrm{NC}$ & Non-responder & Learning \\
\hline KIS-040 & $\mathrm{F}$ & 68 & Clear cell carcinoma & $\mathrm{PD}$ & Non-responder & Learning \\
\hline KIS-046 & M & 51 & Clear cell carcinoma & PD & Non-responder & Learning \\
\hline KIS-048 & M & 67 & Clear cell carcinoma & PD & Non-responder & Learning \\
\hline KIS-049 & $\mathrm{F}$ & 51 & Clear cell carcinoma & $\mathrm{NC}$ & Non-responder & Learning \\
\hline KIS-051 & $\mathrm{M}$ & 73 & Clear cell carcinoma & $\mathrm{NC}$ & Non-responder & Learning \\
\hline KIS-052 & $\mathrm{F}$ & 71 & $\begin{array}{l}\text { Combined type (clear cell, granular cell } \\
\text { and spindle cell carcinoma) }\end{array}$ & $\mathrm{PD}$ & Non-responder & Learning \\
\hline KIS-057 & $\mathrm{F}$ & 60 & Clear cell carcinoma & $\mathrm{PD}$ & Non-responder & Learning \\
\hline KIS-059 & M & 60 & Clear cell carcinoma & $\mathrm{NC}$ & Non-responder & Learning \\
\hline KIS-060 & M & 56 & Clear cell carcinoma & $\mathrm{NC}$ & Non-responder & Learning \\
\hline KIS-066 & M & 61 & Clear cell carcinoma & $\mathrm{NC}$ & Non-responder & Learning \\
\hline KIS-012 & M & 62 & Clear cell carcinoma & PR & Responder & Test \\
\hline KIS-029 & $\mathrm{F}$ & 57 & Clear cell carcinoma & PR & Responder & Test \\
\hline KIS-043 & M & 64 & Clear cell carcinoma & PR & Responder & Test \\
\hline KIS-045 & $\mathrm{M}$ & 62 & Papillary renal cell carcinoma & PR & Responder & Test \\
\hline KIS-065 & $\mathrm{F}$ & 39 & $\begin{array}{l}\text { Combined type (clear cell carcinoma } \\
\text { and spindle cell carcinoma) }\end{array}$ & PR & Responder & Test \\
\hline KIS-003 & $\mathrm{F}$ & 56 & Clear cell carcinoma & $\mathrm{NC}$ & Non-responder & Test \\
\hline KIS-004 & M & 58 & Clear cell carcinoma & MR & Non-responder & Test \\
\hline KIS-015 & M & 40 & Clear cell carcinoma & $\mathrm{NC}$ & Non-responder & Test \\
\hline KIS-023 & $\mathrm{M}$ & 71 & Clear cell carcinoma & MR & Non-responder & Test \\
\hline KIS-030 & $\mathrm{F}$ & 51 & Clear cell carcinoma & $\mathrm{NC}$ & Non-responder & Test \\
\hline KIS-033 & M & 72 & Clear cell carcinoma & $\mathrm{PD}$ & Non-responder & Test \\
\hline KIS-047 & M & 76 & Clear cell carcinoma & MR & Non-responder & Test \\
\hline KIS-056 & M & 69 & Clear cell carcinoma & MR & Non-responder & Test \\
\hline KIS-064 & $\mathrm{M}$ & 61 & Clear cell carcinoma & PD & Non-responder & Test \\
\hline
\end{tabular}

Response, response to IL-2 and IFN- $\alpha$ combination therapy for RCC with lung metastasis; Responder, CR or PR; Non-responder, PD, NC or MR. CR, complete response (shrinkage rate 100\%); PR, partial response (shrinkage rate $\geq 50$ to $<100 \%$ ); MR, minor response (shrinkage rate $\geq 25$ to $<50 \%$ ); NC, no change (shrinkage rate $\geq-25$ to $<25 \%$ ); PD, progressive disease (shrinkage rate $\leq-25 \%$ ). Shrinkage rate, tumor shrinkage rate in pulmonary metastasis. 
Table II. Clinical response and classification of the two groups.

\begin{tabular}{lll}
\hline Group $\quad$ Clinical response No. of patients \\
\hline
\end{tabular}

Responder

$\begin{array}{lr}\text { CR } & 2 \\ \text { PR } & 13\end{array}$

Non-responder

$\begin{array}{lr}\text { MR } & 4 \\ \text { NC } & 12 \\ \text { PD } & 11\end{array}$

Total

Responder group, CR or PR; non-responder group, PD, NC or MR. CR, complete response (shrinkage rate 100\%); PR, partial response (shrinkage rate $\geq 50$ to $<100 \%$ ); MR, minor response (shrinkage rate $\geq 25$ to $<50 \%$ ); NC, no change (shrinkage rate $\geq-25$ to $<25 \%$ ); PD, progressive disease (shrinkage rate $\leq-25 \%$ ). Shrinkage rate, tumor shrinkage rate in pulmonary metastasis. Efficacy rate $35.7 \%$. from patients that had met the inclusion criteria of this clinical trial as reported previously (21). To attempt to establish a prediction method for clinical responses to IL-2 and IFN- $\alpha$ combination therapy, we analyzed gene expression profiles of microdissected renal cancer cells using an oligoDNA microarray consisting of 38,500 genes or ESTs. Analyzing 10 responders (CR or PR) and 18 non-responders (PD or NC), we identified 24 genes that showed significantly different levels of expression between the responder and non-responder groups according to the two following definitions: i) expression information based on signal intensities higher than the cutoff level in $>60 \%$ of samples of at least one group; ii) random permutation test P-values $<0.0005$ (Table III). Among the 24 genes selected, the expression levels of 14 genes were higher in the responder group, including two immunologically important genes, HLA-DQA1 (P=2.83E-05) and HLA-DQB1 $(\mathrm{P}=2.66 \mathrm{E}-04)$, that are known to be HLA class II molecules having critical roles in antigen presentation. Of the remaining 22 genes, three genes have also been implicated in several roles in immunological responses: MAP3K5 (ASK1) is

Table III. List of 24 discriminating genes.

\begin{tabular}{|c|c|c|c|c|}
\hline Accession no. & Symbol & P-value & Sign & Gene title \\
\hline NM_004193 & GBF1 & 8.87E-07 & - & Golgi-specific brefeldin A resistance factor 1 \\
\hline NM_005575 & LNPEP & $1.49 \mathrm{E}-06$ & + & Leucyl/cystinyl aminopeptidase \\
\hline NM_005923 & MAP3K5 & $3.47 \mathrm{E}-06$ & + & Mitogen-activated protein kinase kinase kinase 5 \\
\hline AW452656 & ----- & $6.95 \mathrm{E}-06$ & - & cDNA FLJ37989 fis, clone CTONG2011676 \\
\hline NM_007081 & RABL2B & $1.36 \mathrm{E}-05$ & - & RAB, member of RAS oncogene family-like $2 \mathrm{~B}$ \\
\hline X63381 & MEF2A & $2.26 \mathrm{E}-05$ & + & Myocyte enhancer factor $2 \mathrm{~A}$ \\
\hline AK093779 & LOC399900 & $2.45 \mathrm{E}-05$ & - & Hypothetical gene supported by AK093779 \\
\hline X00452 & HLA-DQA1 & $2.83 \mathrm{E}-05$ & + & Major histocompatibility complex, class II, DQ $\alpha 1$ \\
\hline AK023514 & TDP1 & $3.76 \mathrm{E}-05$ & + & Tyrosyl-DNA phosphodiesterase 1 \\
\hline NM_018835 & $\mathrm{RC} 3 \mathrm{H} 2$ & $3.99 \mathrm{E}-05$ & + & Ring finger and $\mathrm{CCCH}$-type zinc finger domain 2 \\
\hline AL096842 & MTUS1 & 4.05E-05 & + & Mitochondrial tumor suppressor 1 \\
\hline NM_007121 & $\mathrm{NR} 1 \mathrm{H} 2$ & $5.80 \mathrm{E}-05$ & - & Nuclear receptor subfamily 1 , group $\mathrm{H}$, member 2 \\
\hline AA131302 & ----- & $8.88 \mathrm{E}-05$ & - & $\begin{array}{l}\text { Transcribed locus, weakly similar to NP_001039959.1 } \\
\text { dynamin 1-like (Bos taurus) }\end{array}$ \\
\hline NM_003170 & SUPT6H & $1.16 \mathrm{E}-04$ & - & Suppressor of Ty 6 homolog (S. cerevisiae) \\
\hline NM_001190 & BCAT2 & $1.17 \mathrm{E}-04$ & - & Branched chain aminotransferase 2, mitochondrial \\
\hline BF060747 & LOC130576 & $1.20 \mathrm{E}-04$ & + & Hypothetical protein LOC130576 \\
\hline BC000580 & PH-4 & $1.57 \mathrm{E}-04$ & - & Hypoxia-inducible factor prolyl 4-hydroxylase \\
\hline NM_024605 & ARHGAP10 & $1.91 \mathrm{E}-04$ & + & Rho GTPase activating protein 10 \\
\hline BG231758 & ----- & $1.92 \mathrm{E}-04$ & + & $\begin{array}{l}\text { Transcribed locus, strongly similar to NP_001025836.1 } \\
\text { tuftelin interacting protein } 11 \text { (Gallus gallus) }\end{array}$ \\
\hline AB029026 & TACC1 & $2.56 \mathrm{E}-04$ & + & Transforming, acidic coiled-coil containing protein 1 \\
\hline M16276 & HLA-DQB1 & $2.66 \mathrm{E}-04$ & + & Major histocompatibility complex, class II, DQ $\beta 1$ \\
\hline NM_012463 & ATP6V0A2 & $3.51 \mathrm{E}-04$ & + & ATPase, $\mathrm{H}+$ transporting, lysosomal V0 subunit a2 \\
\hline J03225 & TFPI & $3.61 \mathrm{E}-04$ & + & $\begin{array}{l}\text { Tissue factor pathway inhibitor } \\
\text { (lipoprotein-associated coagulation inhibitor) }\end{array}$ \\
\hline AJ011597 & BDNFOS & 4.45E-04 & - & Brain-derived neurotrophic factor opposite strand \\
\hline
\end{tabular}

P-values were calculated by random permutation tests. Information was retrieved from AGCC annotation database (Affymetrix). +, geneincreased expression in the responder group; -, gene-increased expression in the non-responder group. 
A

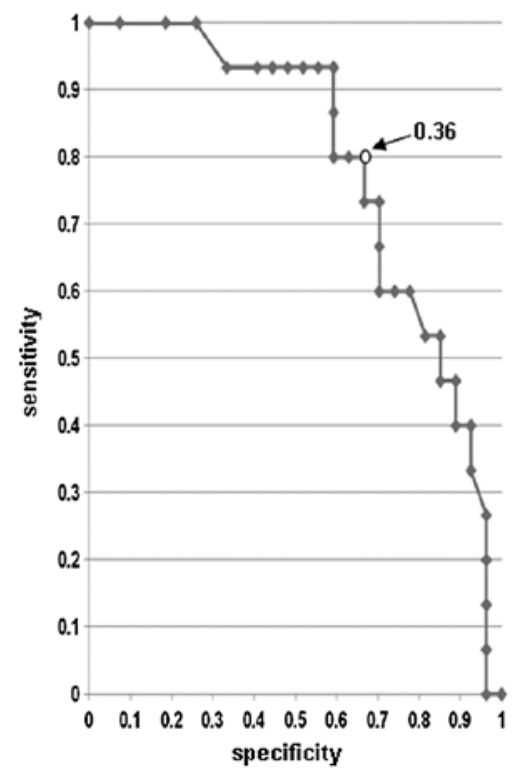

B

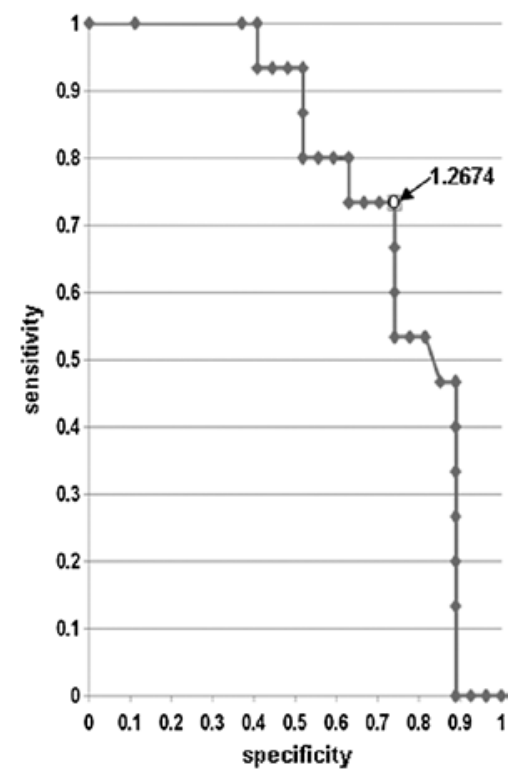

Figure 1. Receiver operating characteristic (ROC) curve. Result of microarray data. Sensitivity shows the ratio of the number of samples for which a value is higher than each cutoff value in the responder group. Specificity shows the ratio of the number of samples for which a value is higher than each cutoff value in the non-responder group. Specificity indicates the number for which the 'ratio of the number of samples for which a value is higher than each cutoff value in the non-responder group' is subtracted from ' 1 '. The point where 'sensitivity' and 'specificity' were both near ' 1 ' [in short where ' $(1 \text { - sensitivity })^{2}+(1 \text { - specificity })^{2}$ ' was the lowest] was determined to be the optimal cutoff value for the expression level of each gene based on the ROC curve. (A) ROC curve of HLA-DQA1. The optimum cutoff value (arrow) was 0.36 ; at this point, sensitivity was 0.80 and specificity was 0.67 . (B) ROC curve of HLA-DQB1. The optimum cutoff value (arrow) was 1.2674; at this point, sensitivity was 0.73 and specificity was 0.74 .

suggested to be related to the natural immunity to the stress response. TFPI is suggested to function as a negative regulator of cytokine expression. In addition, NR4A2 has been identified as a candidate target molecule for the treatment of multiple sclerosis, one of the autoimmune diseases of the central nervous system.

Since IL-2 and IFN- $\alpha$ treatment is expected to enhance patient immunity with the subsequent attack by immune
A

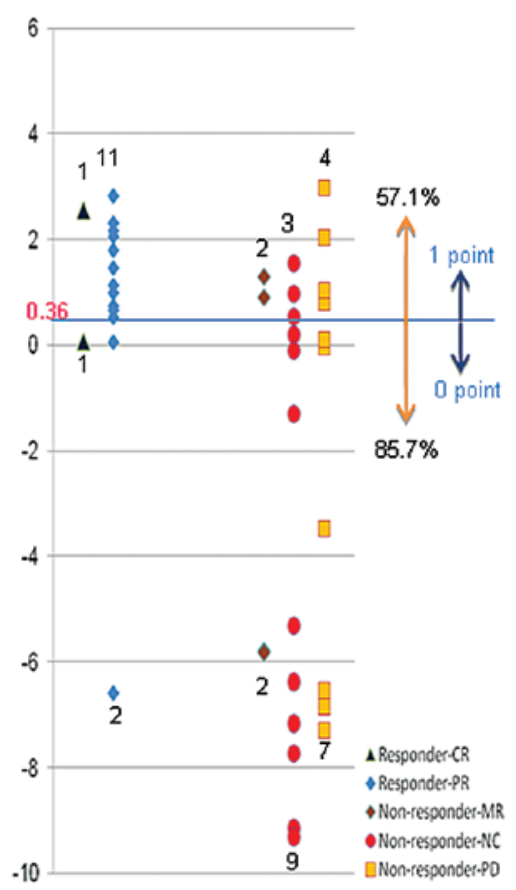

B

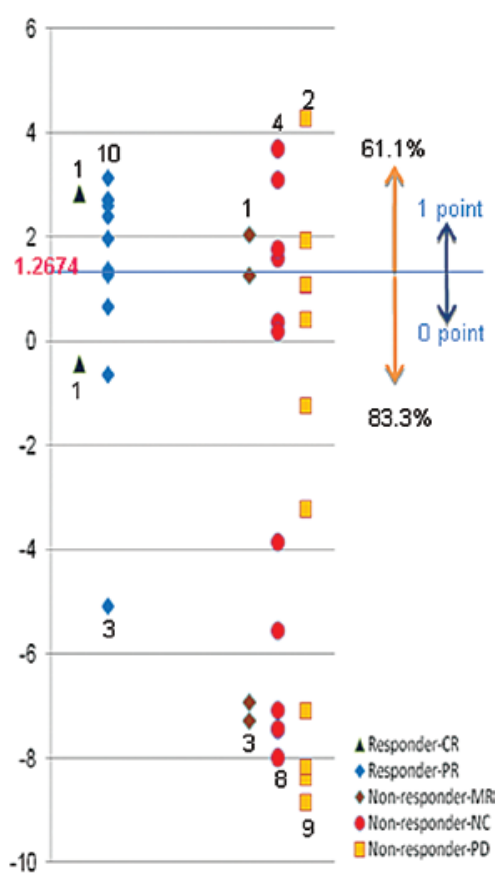

Figure 2. Expression pattern. The figures of the spindle show the value, which was divided by the expression level of the normal tissue and transformed to a logarithm. Dark blue squares, CR; blue diamonds, PR; brown diamonds, MR; red circles, NC; orange squares, PD. Samples whose expression levels were higher than these cutoff values were assigned a score of ' 1 ' and samples whose expression levels were lower than these cutoff values were assigned a score of ' 0 '. (A) Expression pattern of HLA-DQA1. (B) Expression of HLADQB1.

cells on cancer cells, these two HLA class II molecules were considered to likely play key roles in clinical response. Hence, we focused on these two molecules measuring the expression levels quantitatively and comparing them to clinical response. We confirmed the relativity of expression level and clinical response using the receiver operating characteristic (ROC) curve, and the best cutoff value for the expression level of each gene was determined (Fig. 1). The results demonstrated that 
Table IV. Scoring-based expression pattern 1.

\begin{tabular}{lccr}
\hline Group & \multicolumn{3}{c}{ Score } \\
\cline { 2 - 4 } & 0 & 1 & 2 \\
\hline $\begin{array}{l}\text { Non-responder (n=27) } \\
(\mathrm{MR}+\mathrm{PD}+\mathrm{NC})\end{array}$ & 16 & 6 & 5 \\
$\begin{array}{l}\text { Responder }(\mathrm{n}=15) \\
(\mathrm{CR}+\mathrm{PR})\end{array}$ & 2 & 3 & 10 \\
\hline
\end{tabular}

Summary table of the scoring-based expression pattern of Fig. 2. Score: 0, HLA-DQA1 and HLA-DQB1 expression values both less than the cutoff value; 1, HLA-DQA1 (or HLA-DQB1) expression value less than the cutoff value and HLA-DQB1 (or HLA-DQA1) expression value greater than the cutoff value; 2, HLA-DQA1 and HLA-DQB1 expression values both greater than the cutoff value. CR, complete response; $\mathrm{PR}$, partial response; MR, minor response; $\mathrm{NC}$, no change; PD, progressive disease.

Table V. Scoring-based expression pattern 2.

\begin{tabular}{lccr}
\hline Group & \multicolumn{3}{c}{ Score } \\
\cline { 2 - 4 } & 0 & 1 & 2 \\
\hline $\begin{array}{l}\text { Non-responder }(\mathrm{n}=27) \\
(\mathrm{MR}+\mathrm{PD}+\mathrm{NC})\end{array}$ & 16 & 6 & 5 \\
$\begin{array}{l}\text { Responder }(\mathrm{n}=15) \\
(\mathrm{CR}+\mathrm{PR})\end{array}$ & 1 & 4 & 10 \\
\end{tabular}

Summary table of scoring-based expression pattern of quantitative RT-PCR. Score: 0, HLA-DQA1 and HLA-DQB1 expression values both less than the cutoff value; 1, HLA-DQA1 (or HLA-DQB1) expression value less than the cutoff value and HLA-DQB1 (or HLADQA1) expression value greater than the cutoff value; 2, HLA-DQA1 and HLA-DQB1 expression values both greater than the cutoff value. $\mathrm{CR}$, complete response; $\mathrm{PR}$, partial response; $\mathrm{MR}$, minor response; $\mathrm{NC}$, no change; $\mathrm{PD}$, progressive disease.

IL-2 and IFN- $\alpha$ combination therapy showed poor response rates of 85.7 and $83.3 \%$, respectively, when the expression level of HLA-DQA1 or HLA-DQB1 was lower than the cutoff value (Fig. 2). If patients having a lower expression of these genes were excluded, than the rate of effectiveness of this therapy is expected to be $57.1 \%$ in the case of HLA-DQA1 and $61.1 \%$ in the case of HLA-DQB1, respectively. However, since HLA-DQA1 and HLA-DQB1 are known to form a heterodimer in the antigen presentation process, we hypothesized that if either one was expressed lower than the cutoff level, the tumor cells were unlikely to produce an HLA-class II molecule sufficiently and were then unable to present the antigen(s) effectively. Considering this hypothesis, if this treatment was withheld to the patients whose tumors expressed a lower level of either HLA-DQA1 or HLA-DQB1 than each cutoff value, than the response rate could be expected to reach as high as $66.7 \%$ (Fig. 2, Table IV).
A

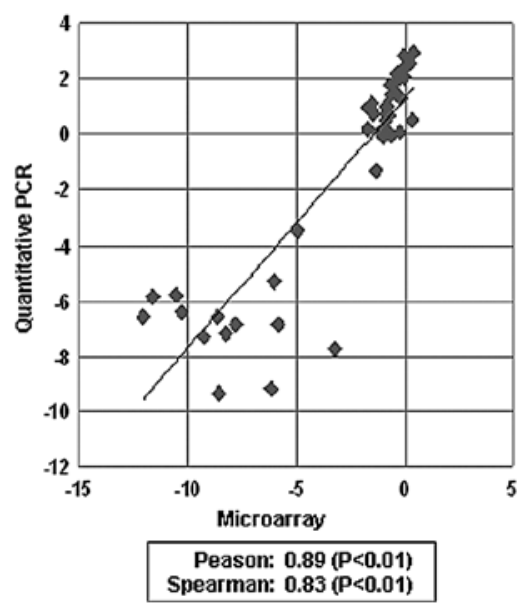

$\mathbf{B}$

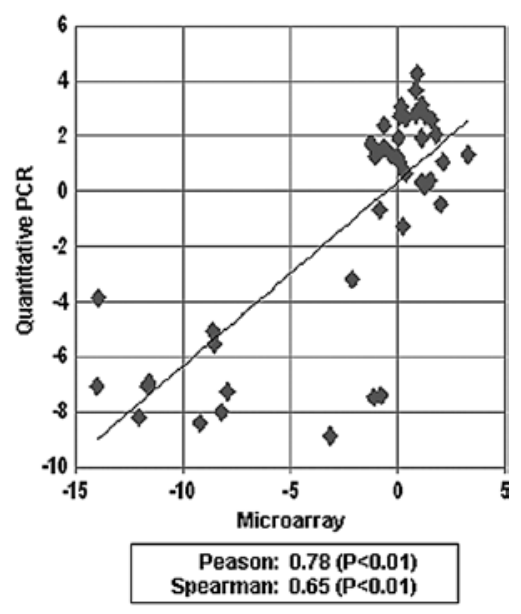

Figure 3. Comparison of microarray data for HLA-DQA1 and HLA-DQB1 with quantitative RT-PCR data. (A) Correlation of microarray data and realtime PCR for HLA-DQA1. (B) Correlation of microarray data and real-time PCR for HLA-DQB1.

\section{Discussion}

To screen genes that may be associated with the clinical response of advanced RCCs to IL- 2 and IFN- $\alpha$ combination therapy, oligo DNA microarray analysis was applied in combination with LMM to obtain precise expression profile data of thousands of genes simultaneously. The following random permutation test of expression data identified 24 candidate genes that exhibited significant differential expression between the 'responder' and 'non-responder' groups (Table III). Since two immunologically important genes, HLA-DQA1 and HLA-DQB1, were included in the set of 24 genes, the relative expression levels were measured and then compared to clinical responses by applying the ROC curve for these two genes (Figs. 1 and 2; Table IV). As HLA-DQA1 and HLA-DQB1 are known to form a heterodimer, we hypothesized that when either gene exhibited a low level of expression in tumors, the antigen presentation from the tumor cells for immunotherapy may be insufficient. When such patients were excluded from this particular treatment, the rate of effectiveness of this therapy was expected to improve to $65-70 \%$ (Table IV).

To further apply our prediction system in clinical use, we attempted to establish the quantitative RT-PCR method. As a result, the mRNA levels measured by the quantitative 
RT-PCR method were found to be quite consistent to those obtained from the microarray analysis with Pearson's correlation coefficient and Spearman's rank-correlation coefficient of 0.89 and 0.83 for HLA-DQA1, respectively, and 0.78 and 0.65 for HLA-DQB1, respectively (Table V, Fig. 3).

In conclusion, the expression levels of HLA-DQA1 and HLA-DQB1 are reliable candidate markers for predicting the response to IL-2 and IFN- $\alpha$ combination therapy for RCC and provide useful information for the establishment of personalized treatment.

\section{Acknowledgements}

We thank Ms. Noriko Sudo for the data and sample management, Ms. Noriko Ikawa for the preparation of the samples by cryostat, Ms. Kyoko Kijima for the preparation of materials and Drs Yataro Daigo, Koichi Matsuda, Ryuji Hamamoto, Ryo Takata, Chikako Fukukawa, Yosuke Harada, Jae-Hyun Park, Masahiro Ajiro, Suyoun Chung, Akira Togashi, Yasuo Mochiduki, Shinya Hayami, Cho Hyuu-Soo, Goji Toyokawa, Masashi Takawa, Tadashi Kizaki, Mitsuko Nakashima and Kazuma Kiyotani for the helpful discussion and comments. This study was supported by Shionogi \& Co., Ltd.

\section{References}

1. Ferlay J, Bray F, Pisani P and Parkin DM: GLOBOCAN 2002: Cancer Incidence, Mortality and Prevalence Worldwide. IARC CancerBase No. 5, Version 2.0. IARC Press, Lyon, 2004.

2. Parkin DM, Bray F, Ferlay J and Pisani P: Global cancer statistics, 2002. CA Cancer J Clin 55: 74-108, 2005.

3. Motzer RJ, Dror Michaelson M, Redman BG, et al: Activity of SU11248, a multitargeted inhibitor of vascular endothelial growth factor receptor and platelet-derived growth factor receptor, in patients with metastatic renal cell carcinoma. J Clin Oncol 24: 16-24, 2006.

4. Motzer RJ, Rini BI, Bukowski RM, et al: Sunitinib in patients with metastatic renal cell carcinoma. JAMA 295: 2516-2524, 2006.

5. Motzer RJ, Hutson TE, Tomczak P, et al: Sunitinib versus interferon alpha in metastatic renal-cell carcinoma. N Engl J Med 356: 115-124, 2007.

6. Escudier B, Eisen T, Stadler WM, et al: Sorafenib in advanced clear-cell renal-cell carcinoma. N Engl J Med 356: 125-134, 2007.

7. Szcylik C, Demkow T, Staehler M, et al: Randomized phase II trial of first-line treatment with sorafenib versus interferon in patients with advanced renal cell carcinoma: final results. J Clin Oncol 25: 5025, 2007.
8. Aso Y, Tazaki H, Umeda T and Marumo K: A phase II study of S-6820 (recombinant interleukin-2) on renal cell carcinoma (in Japanese). Biotherapy 3: 999-1007, 1989.

9. Aso Y, Homma Y, Tazaki H, et al: A phase II trial of S-6820 (recombinant interleukin-2) on renal cell carcinoma refractory to interferon (in Japanese). Hinyokigeka 8: 75-86, 1995.

10. Hayashi T, Miyagawa Y, Tsujimura A, Nonomura N, Minami M and Okuyama A: A case of renal cell carcinoma with multiple lung metastases refractory to interferon- $\alpha$ showing complete remission by interleukin-2 monotherapy. Int J Urol 13: 805-808, 2006.

11. Kobayashi M, Ikeda H, Nukui A, et al: Clinical outcome and prognostic survival factors in patients with advanced renal cell carcinoma treated with very low-dose interleukin- 2 , interferon- $\alpha$ and tegafur-uracil: a single-institution experience. Int $\mathrm{J}$ Clin Oncol 13: 257-262, 2008.

12. Wirth MP: Immunotherapy for metastatic renal cell carcinoma. Urol Clin North Am 20: 283-295, 1993.

13. Horoszewicz JS and Murphy GP: An assessment of the current use of human interferons in therapy of urological cancers. J Urol 142: 1173-1180, 1989.

14. Bukowski RM: Natural history and therapy of metastatic renal cell carcinoma: the role of interleukin-2. Cancer 80: 1198-1220, 1997.

15. Motzer RJ and Russo P: Systemic therapy for renal cell carcinoma. J Urol 163: 408-417, 2000.

16. Klapper JA, Downey SG, Smith FO, et al: High-dose interleukin-2 for the treatment of metastatic renal cell carcinoma: a retrospective analysis of response and survival in patients treated in the Surgery Branch at the National Cancer Institute between 1986 and 2006. Cancer 113: 293-301, 2008.

17. Belldegrun AS, Klatte T, Shuch B, et al: Cancer-specific survival outcomes among patients treated during the cytokine era of kidney cancer (1989-2005): a benchmark for emerging targeted cancer therapies. Cancer 113: 2457-2463, 2008.

18. Akaza H, Tsukamoto T, Onishi T, Miki T, Kinouchi T and Naito S: A low-dose combination therapy of interleukin- 2 and interferon- $\alpha$ is effective for lung metastasis of renal cell carcinoma: a multicenter open study. Int J Clin Oncol 11: 434-440, 2006.

19. Akaza H, Kawai K, Tsukamoto T, et al: Successful outcomes using combination therapy of interleukin- 2 and interferon- $\alpha$ for renal cell carcinoma patients with lung metastasis. Jpn J Clin Oncol 40: 684-689, 2010.

20. WHO Handbook for Reporting Results of Cancer Treatment. WHO Offset Publication No. 48. World Health Organization, Geneva, 1979.

21. Japanese Urological Association and Japanese Society of Pathology: Response criteria for urological cancer treatment. Jpn J Urol 83: 447-472, 1992.

22. Golub TR, Slonim DK, Tamayo P, et al: Molecular classification of cancer: class discovery and class prediction by gene expression monitoring. Science 286: 531-537, 1999. 